\title{
Cardiac magnetic resonance imaging characterizes stenosis, perfusion, and fibrosis preoperatively and postoperatively in children with anomalous coronary arteries
}

\author{
Julie A. Brothers, MD, ${ }^{a}$ Timothy S. Kim, BA, ${ }^{a}$ Mark A. Fogel, MD, ${ }^{a, b}$ Kevin K. Whitehead, MD, PhD, ${ }^{a, b}$ \\ Tonia M. Morrison, MSM, CCRC, ${ }^{a}$ Stephen M. Paridon, MD, ${ }^{a}$ and Matthew A. Harris, MD ${ }^{\mathrm{a}, \mathrm{b}}$
}

\section{ABSTRACT}

Objectives: Using cardiac magnetic resonance imaging (MRI) with virtual angioscopy, we sought to evaluate coronary anatomy, myocardial injury, and left ventricular function in children with interarterial anomalous aortic origin of coronary artery before and after surgery.

Methods: We prospectively enrolled children 5 to 19 years old with interarterial anomalous coronary artery. Cardiac MRI was performed with respiratorynavigated steady-state free-precession 3-dimensional data set acquisition. Virtual angioscopy was used to evaluate the coronary ostia directly. Surgery consisted of the modified unroofing procedure.

Results: We enrolled 9 subjects between February 2009 and May 2015. Subjects were male, with an average age at surgery of 14.1 years. Anomalous coronary was right in 7 patients $(77 \%)$ and left in 2 . In all subjects, the proximal anomalous coronary arose tangential to the aorta with an elliptical, slitlike ostium. Before the operation, the proximal coronary artery was significantly smaller proximally than distally ( 2 vs $3.1 \mathrm{~mm} ; P<.0001$. After the operation, neo-orifices were round and patent in 7 subjects; however, 2 subjects still had narrowed neo-orifices. New postoperative MRI findings included flattened septal wall motion $(\mathrm{N}=1)$, small region of midmyocardial partial-thickness scar $(\mathrm{N}=1)$, and dyskinetic septal wall motion with mild aortic insufficiency $(\mathrm{N}=1)$. Left ventricular function was normal both before and after surgery $(P=.85)$.

Conclusions: Cardiac MRI with virtual angioscopy is an important tool for evaluating anomalous coronary anatomy, myocardial function, and ischemia and should be considered for initial and postoperative assessment of children with anomalous coronary arteries. (J Thorac Cardiovasc Surg 2016;152:205-10)

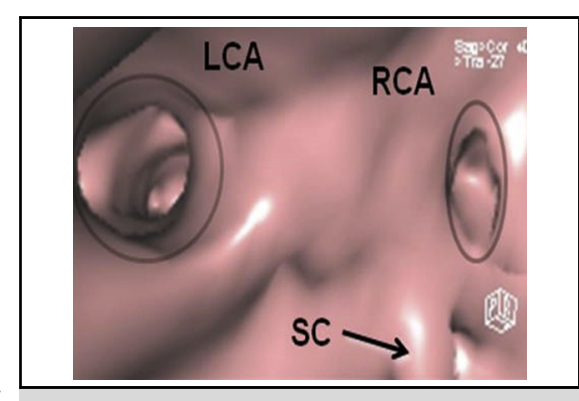

After surgery, RCA is narrow; virtual angioscopy shows the neo-ostium remains elliptical.

\section{Central Message}

Cardiac MRI with virtual angioscopy should be used for initial and postoperative assessment in youths with anomalous coronary arteries.

\section{Perspective}

The ostia in children with anomalous coronary arteries are slitlike at diagnosis but after surgery are generally round and unobstructed; however, 2 subjects' neo-ostia remained elliptical and somewhat stenotic after surgery. The significance of these findings is unknown, but a continued stenotic orifice may place subjects at risk for future sudden death, necessitating close long-term follow-up.

See Editorial Commentary page 211
Anomalous aortic origin of a coronary artery that courses between the 2 great vessels (AAOCA) is a rare congenital cardiac anomaly that may carry an increased risk for myocardial ischemia and sudden cardiac death in young

\footnotetext{
From the ${ }^{\mathrm{a}}$ Division of Cardiology, Department of Pediatrics, and the ${ }^{\mathrm{b}}$ Department of Radiology, The Children's Hospital of Philadelphia and Perelman School of Medicine at the University of Pennsylvania, Philadelphia, Pa.

Supported by the American Heart Association (grant No. 11SDG4910008) and The Cardiac Center at The Children's Hospital of Philadelphia.

Received for publication Oct 28, 2015; revisions received Dec 18, 2015; accepted for publication Dec 24, 2015; available ahead of print Feb 6, 2016.

Address for reprints: Julie A. Brothers, MD, Division of Cardiology, 34th and Civic Center Blvd, 8th Floor, Philadelphia, PA 19104 (E-mail: brothersj@email.chop.edu). $0022-5223 / \$ 36.00$

Copyright (c) 2016 by The American Association for Thoracic Surgery http://dx.doi.org/10.1016/j.jtcvs.2015.12.057
}

people. $^{1-3}$ The initial diagnosis of AAOCA is usually made by transthoracic echocardiography. Most pediatric patients also undergo confirmatory tests consisting of cardiac MRI or computed tomographic imaging. It is important to delineate the coronary anatomy accurately, including the ostium, because the collapse of an elliptical and stenotic ostium with exertion has been proposed as a possible mechanism of sudden death in patients with

Scanning this QR code will take you to the article title page.

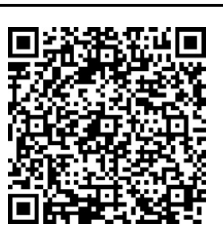




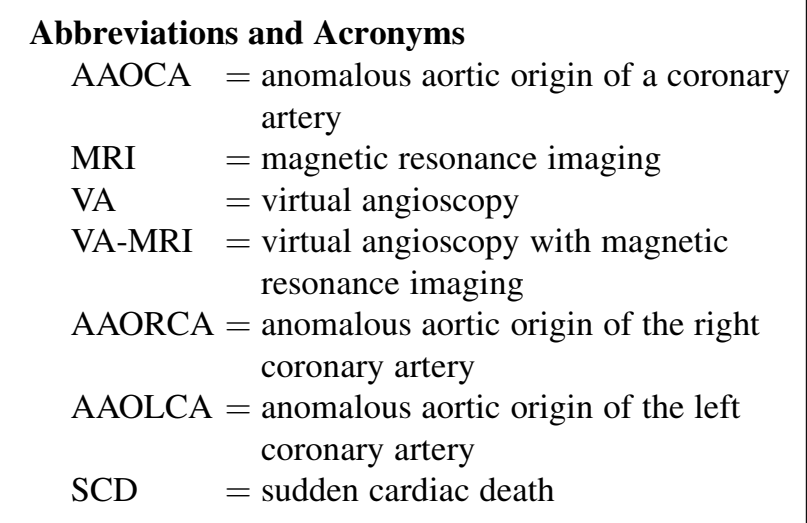

interarterial, intramural AAOCA. MRI can be used to evaluate for ostial stenosis, especially when done with virtual angioscopy (VA) analysis. VA is a technique that enables the observer to view the anatomy of interest as if standing within the vessel itself. By using MRI with VA (VA-MRI), we sought to compare the shape and size of the coronary ostia, evidence of myocardial injury, and left ventricular function before and after surgical repair for AAOCA in the pediatric population.

\section{MATERIALS AND METHODS}

Study approval was obtained from the institutional review board committee at The Children's Hospital of Philadelphia. After consent had been obtained, we prospectively enrolled subjects 5 to 19 years old with AAOCA who were scheduled for surgical repair. We performed a chart review on each subject. Subjects underwent preoperative and postoperative VA-MRI at The Children's Hospital of Philadelphia between February 2009 and May 2015. We used descriptive statistics and paired $t$ tests to evaluate for statistical significance.

\section{Cardiac MRI Protocol}

The children underwent a VA-MRI in the Division of Radiology at The Children's Hospital of Philadelphia. Each study was performed with respiratory-navigated, steady-state free-precession 3-dimensional data set acquisition with a 1.5-T clinical MRI scanner (Siemens Avanto 1.5T; Siemens Medical Systems, Malvern, Pa). T1-weighted gradient echo imaging of 2 adjacent left ventricular slices in the short-axis orientation, with a non-slice-selective saturation recovery magnetization preparation, with the temporal GeneRalized Autocalibrating Partial Parallel Acquisition procedure used for times 2 acceleration, was used to image the first pass of injected contrast bolus through the heart. A bolus of MRI contrast agent (Magnevist; Berlex Laboratories, Inc, Wayne, NJ) at a dosage of $0.04 \mathrm{mmol} / \mathrm{kg}$ of body weight was administered through an antecubital vein, starting at the third or fourth heart beat, with a power injector at a rate of $4 \mathrm{~mL} / \mathrm{s}$, followed by a saline solution flush of $15 \mathrm{~mL}$ at the same injection rate. Myocardial delayed enhancement images were performed 10 minutes after gadolinium administration in 4-chamber long-axis and short-axis views of the left ventricle.

Preoperative and postoperative VA-MRI studies were reviewed to determine each coronary artery sinus of origin, proximal course, left ventricular dimensions, and cardiac function. We used multiplanar reconstruction software, which allows reformatting of the 3dimensional data set to produce any desired 2-dimensional slice plane.

\section{Virtual Angioscopy}

VA was used to evaluate the coronary ostia directly and determine their proximity to the intercoronary commissure. The VA software is commercially available and resident on a Leonardo satellite workstation (Siemens Corp, New York, NY) in our Department of Radiology. The VA technique has been previously described for the evaluation of coronary arteries, ${ }^{4,5}$ and it has been recently validated by our group..$^{5}$ By means of VA, the 3dimensional coronary imaging data set was evaluated as if viewed from within the aortic sinuses, simulating the perspective from which a surgeon or pathologist would observe the origin of the coronary artery. The VA "camera" is positioned in the center of the aorta and focused perpendicular to the plane of the ostium to assess for ostial shape. Thresholding was performed to yield a solid aortic wall and optimal contrast for the ostium. VA requires a high-quality 3-dimensional data set for visualization of coronary ostia. The data set can then be analyzed from any perspective in assessing for coronary ostial stenosis. The ostium can be identified as round and unobstructed versus crescentic and stenotic. In addition, the position of the ostium could be viewed relative to the septal commissure to assess whether the ostium is juxtacommisural. The ostia were identified as round versus elliptical, and the ratio of the vertical to the horizontal axis was measured to determine the elliptical nature of the anomalous, normal, and repaired ostia.

\section{Surgical Procedure}

In all subjects, surgery consisted of the modified unroofing procedure. This is a technique used to address the slitlike orifice and intramural segment of the anomalous coronary artery. The anomalous coronary ostium is opened longitudinally, starting at the anomalous coronary os and continuing into the correct sinus. A segment of the common wall between the aorta and coronary artery is excised, and the intimal surfaces are approximated with interrupted sutures. Detachment of the aortic valve commissure combined with resuspension is sometimes necessary. ${ }^{6,7}$

\section{RESULTS}

From February 2009 through May 2015, we enrolled 9 subjects. Of these, $7(77.7 \%)$ had anomalous aortic origin of the right coronary from the left sinus of Valsalva (AAORCA) with an interarterial course; the other 2 had interarterial anomalous aortic origin of the left coronary from the right aortic sinus (AAOLCA). All subjects were male, with a mean age of 14.3 (median of 14.7) years at the time of surgery. All but 1 of the subjects had signs or symptoms referable to the cardiovascular system (Table 1). All subjects had confirmation of slitlike orifices and an intramural course by inspection in the operating room. The modified unroofing procedure was performed on all subjects; 2 subjects also underwent commissural detachment and resuspension. All subjects underwent preoperative and postoperative VA-MRI. The postoperative studies were performed an average of 8.6 (median of 6.5) months after the operation.

Before the operation, the proximal anomalous coronary artery's course was described as "tangential" to the aorta in all subjects. The average size of the proximal coronary 
TABLE 1. Characteristics of patients with anomalous coronary arteries

\begin{tabular}{|c|c|c|c|}
\hline Characteristics & $\operatorname{AAORCA}(\mathbf{N}=7)$ & $\operatorname{AAOLCA}(\mathbf{N}=2)$ & Total $(\mathbf{N}=\mathbf{9})$ \\
\hline $\operatorname{Age}^{*}(\mathrm{y}$, mean $\pm \mathrm{SD})$ & $13.8 \pm 2.5$ & $16 \pm 4.2$ & $14.3 \pm 2.8$ \\
\hline Male & 7 & 2 & 9 \\
\hline \multicolumn{4}{|l|}{ Signs and symptoms } \\
\hline Exertional chest pain & $3(33 \%)$ & 0 & 3 \\
\hline Palpitations and family history of AAORCA & $1(11 \%)$ & 0 & 1 \\
\hline Vasovagal syncope & $1(11 \%)$ & $1(11 \%)$ & 2 \\
\hline Hypotension & $1(11 \%)$ & 0 & 1 \\
\hline ST-segment changes on stress electrocardiography & 0 & $1(11 \%)$ & 1 \\
\hline \multicolumn{4}{|l|}{ Asymptomatic } \\
\hline Heart murmur & $1(11 \%)$ & 0 & 1 \\
\hline
\end{tabular}

Values represent number and percentage unless noted. AAORCA, Anomalous right coronary artery; $A A O L C A$, anomalous left coronary artery; $S D$, standard deviation. *At time of surgery.

artery before the operation was significantly smaller at $2.0 \mathrm{~mm}$, compared with $3.1 \mathrm{~mm}$ distally $(P<.0001)$. According to preoperative VA, all 9 subjects had elliptical, slitlike orifices (Figure 1). After the operation, the neo-orifices in 7 subjects were round and patent without evidence of obstruction (Figure 2); however, 2 subjects with AAORCA had neo-orifices that were still narrow (Figure 3). One 13-year-old subject's neo-ostium was located in the correct aortic sinus after the operation but did not appear round; the proximal right coronary artery remained relatively smaller than the distal right coronary artery (measuring $2 \mathrm{~mm}$ proximally both before and after the operation vs $2.6 \mathrm{~mm}$ distally). One 15-year-old subject's neo-ostium, also in the correct sinus, measured narrow in the superior-inferior dimension. Although it was somewhat larger than it had been before the operation ( $2.2 \mathrm{vs} 1.5 \mathrm{~mm}$ ), the orifice was

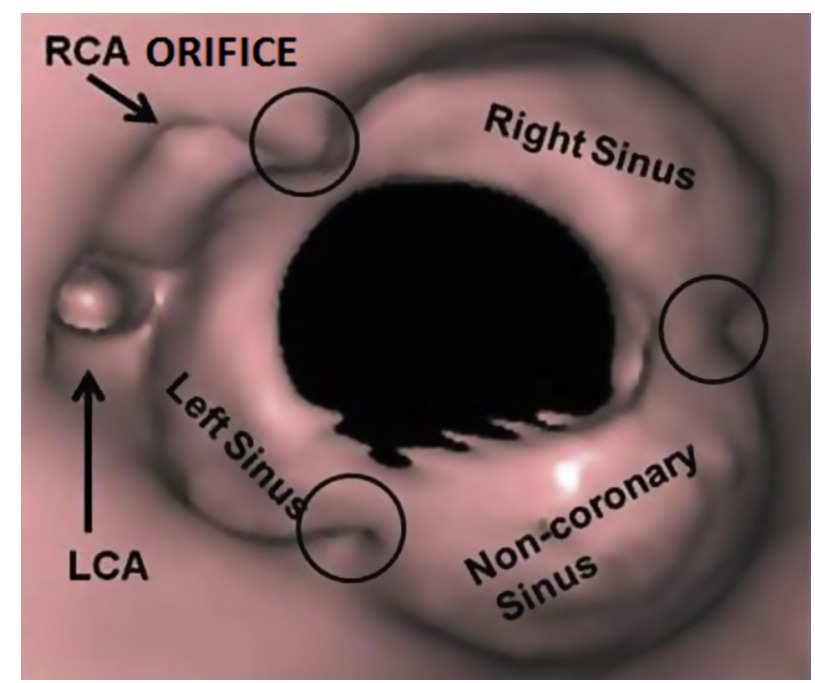

FIGURE 1. Virtual angioscopic view of the aortic sinuses, commissures (circled), and coronary ostia. Note the relatively round appearance of the left coronary artery $(L C A)$, in contrast to the slitlike appearance of the right coronary artery $(R C A)$. still smaller than the distal measurement $(3.5 \mathrm{~mm})$. During this subject's operation, the coronary artery appeared small to the surgeon after unroofing. A patch ostioplasty was performed, but the coronary origin still appeared slitlike; the pericardial patch was therefore taken down and reinserted after an incision was extended onto the coronary itself, with improvement noted in the ostial shape.

Other cardiac findings on preoperative VA-MRI included borderline apical left ventricular noncompaction with mild apical dyskinesis $(\mathrm{N}=1$; AAORCA), marked delayed enhancement in the subendocardial left ventricular posterior basal wall with localized wall thinning and flattened septal wall motion $(\mathrm{N}=1$; AAORCA; Figure 4$)$, and mild mitral regurgitation $(\mathrm{N}=1$; AAORCA; regurgitant fraction $4 \%$ ). Significant postoperative findings not seen before repair included the following: flattened septal wall motion $(\mathrm{N}=1$; AAORCA; 16 months after repair; same subject with borderline noncompaction), small region of midmyocardial partial thickness scar in left ventricular posterior free wall $(\mathrm{N}=1$; AAORCA; 9 months after the operation), dyskinetic septal wall motion and mild aortic insufficiency with regurgitant fraction $5 \%(\mathrm{~N}=1$; AAOLCA; 14 months after the operation). None of these postoperative findings were in the 2 subjects with continued narrowed ostia.

Left ventricular function was normal both before and after surgery (mean ejection fractions of $68.1 \%$ and $67.5 \%$, respectively; $P=.85$ ). Postoperative exercise tests were performed in all subjects at least 3 months after surgery without evidence of new myocardial ischemia since the procedure.

\section{DISCUSSION}

By means of VA-MRI, we demonstrated that the neoostium in children with AAOCA was generally round and unobstructed after the unroofing procedure for AAOCA, in contrast to the presurgical presentation, where the ostium was eccentric, slitlike, and small. Of importance, however, 


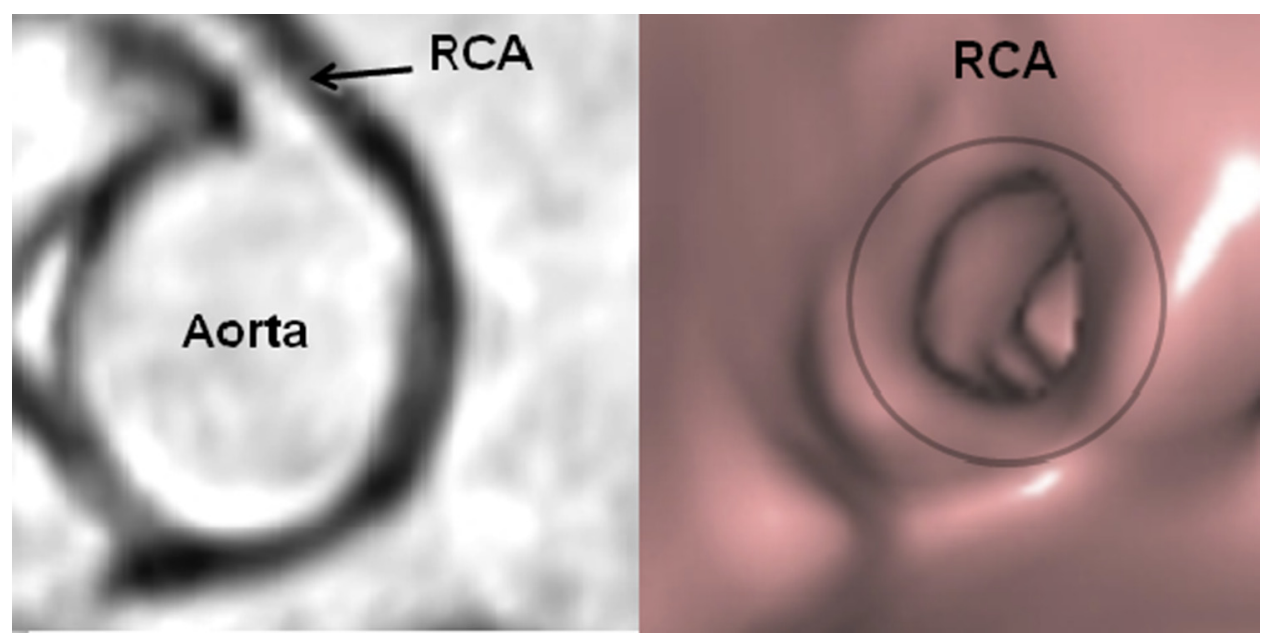

FIGURE 2. After unroofing repair for anomalous right coronary artery $(R C A)$ arising from the left sinus of Valsalva. Note in the left picture that the neoostium originates straight anterior from the right sinus. The virtual angioscopic image on the right confirms a round and patent ostium.

we found that after surgical repair 2 subjects' neo-ostia remained elliptical and somewhat stenotic, and they did not appear round like the corresponding left main coronary artery. The significance of these findings is unknown; howev$\mathrm{er}$, if the proposed mechanism of ischemia is due to a slitlike orifice, a continued stenotic orifice may place subjects at risk for sudden death. To date, these 2 subjects have been free of symptoms, without evidence of ischemia with exercise stress testing or cardiac MRI. Although there are no plans for reintervention, if any concern regarding new evidence of ischemia arises, surgical intervention will need to be considered. We also noted new postoperative cardiac findings, including a midmyocardial scar, dyskinetic wall motion, and mild aortic regurgitation, which were not present before surgery. These subjects will need to be followed up in the future to monitor for progression or resolution.

Documented myocardial ischemia or sudden cardiac death (SCD) from AAOCA in young persons is significantly more common with AAOLCA, but there are rare instances where sudden death has occurred with AAORCA. ${ }^{1-3,8,9}$ SCD is much more likely to occur during or just after vigorous exertion. ${ }^{9-11}$ Although the mechanism for SCD is not known, one postulated cause is that the acute angle takeoff from the aorta creates an elliptical, slitlike orifice that may occlude, notably during exercise when the aorta expands in response to increased stroke volume and wall stress. This may lead to a lethal ventricular arrhythmia. Ischemia is unlikely to occur every time the patient exercises $^{12}$; rather, it appears the collective effect of several
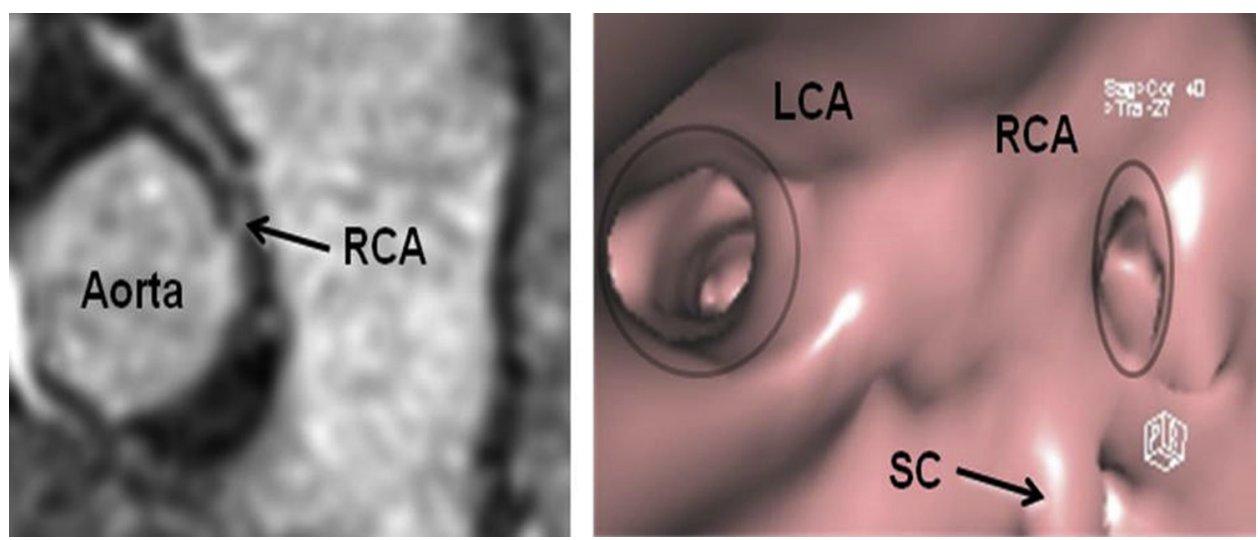

FIGURE 3. A 15-year-old male patient with anomalous right coronary artery $(R C A)$ arising from the left sinus of Valsalva. After the unroofing procedure, the origin still appears far leftward and narrow on the bright blood coronary acquisition. The virtual angioscopic image demonstrates that although the right coronary artery origin now appears slightly to the right of the septal commissure (SC), the ostium remains elliptical and stenotic. The left main coronary artery $(L C A)$ has a round appearance. 


\section{Anomalous RCA Delayed Enhancement}

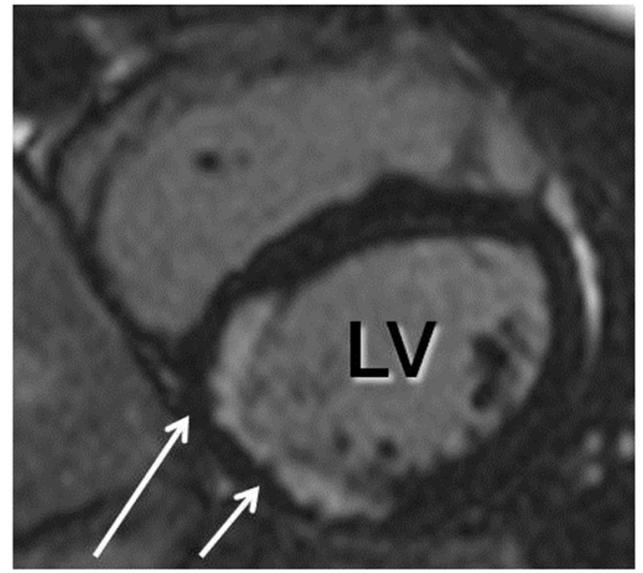

Fibrosis

FIGURE 4. A 15 -year-old male patient with anomalous right coronary artery $(R C A)$ and syncope. Preoperative delayed enhancement imaging after administration of gadolinium contrast agent revealed a subendocardial inferior wall region of left ventricular $(L V)$ enhancement, consistent with fibrosis.

smaller ischemic events may lead to myocardium more prone to lethal tachyarrhythmias. ${ }^{9}$

Patients undergo surgery, most commonly the modified unroofing procedure ${ }^{13,14}$ with the belief that this relieves the coronary anomaly and the subsequent risk of SCD. Because one of the postulated theories of sudden death relates to the shape and size of the ostia, the ability to visualize the orifice and proximal course is potentially critical in risk stratification. Without evidence of a slitlike orifice and presumed intramural course, surgery would not generally be recommended. In addition, if surgery is being performed and children are being cleared for competitive sports after surgery, it is equally important to visualize the postoperative anatomy in detail, which can be accomplished with VA-MRI.

For patients such as those in our cohort, for whom surgery was recommended, the goal is to relieve the potential risk of myocardial ischemia and SCD. When the proximal coronary artery takes an intramural course within the aortic wall, the ostium and proximal coronary artery narrow. The preferred technique for relieving the resultant ostial stenosis and proximal coronary narrowing is the modified unroofing procedure, ${ }^{6,7}$ which addresses what is believed to be the underlying cause of the ischemia, ostial stenosis and intramural course.

Postoperative management generally is composed of electrocardiography and echocardiography. At approximately 3 postoperative months, patients are allowed to return to competitive sports if an exercise stress test shows no evidence of ischemia or significant arrhythmias. ${ }^{15}$ Nguyen and associates, ${ }^{16}$ however, reported the sudden death of a teenaged athlete 2 months after the unroofing procedure; this young man initially was seen with aborted $\mathrm{SCD}$. It may be necessary to alter the activity recommendations for those patients whose presentation includes myocardial infarction or sudden cardiac arrest, because these patients may represent a subset of young people with AAOCA who remain at increased risk of SCD after surgery. At the very least, additional imaging, such as with VA-MRI, should be used in this population. For those patients who are found to have continued narrowing of the neo-ostium, consideration for continued activity restriction from competitive athletics may be indicated, along with closer long-term follow-up.

\section{Limitations}

Study limitations include a small sample size; however, this is a rare disease for which we infrequently perform surgery at our institution. A second potential limitation is associated with the VA imaging. A suboptimal 3-dimensional electrocardiogram and respiratory-gated MRI coronary data set with image blurring will not produce high-quality VA images for assessing the ostium as a whole. For example, images would not be high quality when examining a patient who is noncompliant, has irregular breathing, or has arrhythmia. The images obtained in this study were acquired in an institution very familiar with pediatric cardiac coronary MRI and would be appropriate for assessing the coronary ostia with VA. A final limitation is the lack of long-term follow-up. It will be important to follow these children into adulthood to determine whether any of these abnormalities predispose them toward future increased risk of SCD.

\section{CONCLUSIONS}

This is the first study to document in detail anomalous coronary artery anatomy both before and after surgery. We believe that cardiac MRI is an important tool for the evaluation of anatomy, myocardial function, and ischemia or injury and should be considered for the initial and, when applicable, postoperative assessment of children with AAOCA.

\section{Conflict of Interest Statement}

Authors have nothing to disclose with regard to commercial support.

\section{References}

1. Taylor AJ, Byers JP, Cheitlin MD, Virmani R. Anomalous right or left coronary artery from the contralateral coronary sinus: "high risk" abnormalities in the initial coronary artery course and heterogeneous clinical outcomes. Am Heart J. 1997; 133:428-35. 
2. Roberts WC, Siegel RJ, Zipes DP. Origin of the right coronary artery from the left sinus of Valsalva and its functional consequences: analysis of 10 necropsy patients. Am J Cardiol. 1982;49:863-8.

3. Frescura C, Basso C, Thiene G, Corrado D, Pennelli T, Angelini A, et al. Anomalous origin of coronary arteries and risk of sudden death: a study based on an autopsy population of congenital heart disease. Hum Pathol. 1998;29:689-95.

4. Brothers JA, Whitehead KK, Keller MS, Fogel MA, Paridon SM, Weinberg PM, et al. Cardiac MRI and CT: differentiation of normal ostium and intraseptal course from slit-like ostium and interarterial course in anomalous left coronary artery in children. AJR Am J Roentgenol. 2015;204:W104-9.

5. Harris MA, Whitehead KK, Shin DC, Keller MS, Weinberg PM, Fogel MA. Identifying abnormal ostial morphology in anomalous aortic origin of a coronary artery. Ann Thorac Surg. 2015;100:174-9.

6. Gulati R, Reddy VM, Culbertson C, Helton G, Suleman S, Reinhartz O, et al Surgical management of coronary artery arising from the wrong coronary sinus, using standard and novel approaches. J Thorac Cardiovasc Surg. 2007;134: 1171-8.

7. Moodie DS, Gill C, Loop FD, Sheldon WC. Anomalous left main coronary artery originating from the right sinus of Valsalva: pathophysiology, angiographic definition, and surgical approaches. J Thorac Cardiovasc Surg. 1980;80:198-205.

8. Cheitlin MD, De Castro CM, McAllister HA. Sudden death as a complication of anomalous left coronary origin from the anterior sinus of Valsalva. Circulation. 1974:50:780-7.

9. Basso C, Maron BJ, Corrado D, Thiene G. Clinical profile of congenital coronary artery anomalies with origin from the wrong aortic sinus leading to sudden death in young competitive athletes. J Am Coll Cardiol. 2000;35:1493-501.

10. Maron BJ, Doerer JJ, Haas TS, Tierney DM, Mueller FO. Sudden deaths in young competitive athletes: analysis of 1866 deaths in the United States, 1980-2006. Circulation. 2009; 119:1085-92.
11. Maron BJ, Haas TS, Ahluwalia A, Rutten-Ramos SC. Incidence of cardiovascular sudden deaths in Minnesota high school athletes. Heart Rhythm. 2013;10: 374-7.

12. Brothers J, Carter C, McBride M, Spray T, Paridon S. Anomalous left coronary artery origin from the opposite sinus of Valsalva: evidence of intermittent ischemia. J Thorac Cardiovasc Surg. 2010;140:e27-9.

13. Poynter JA, Williams WG, McIntyre S, Brothers JA, Jacobs ML; Congenital Heart Surgeons' Society AAOCA Working Group. Anomalous aortic origin of a coronary artery: a report from the Congenital Heart Surgeons Society Registry. World J Pediatr Congenit Heart Surg. 2014;5:22-30.

14. Poynter JA, Bondarenko I, Austin EH, DeCampli WM, Jacobs JP, Ziemer G, et al; Congenital Heart Surgeons' Society AAOCA Working Group. Repair of anomalous aortic origin of a coronary artery in 113 patients: a Congenital Heart Surgeons' Society Report. World J Pediatr Congenit Heart Surg. 2014; 5:507-14.

15. Graham TP Jr, Driscoll DJ, Gersony WM, Newburger JW, Rocchini A, Towbin JA. Task force 2: congenital heart disease. J Am Coll Cardiol. 2005; 45:1326-33.

16. Nguyen AL, Haas F, Evens J, Breur JM. Sudden cardiac death after repair of anomalous origin of left coronary artery from right sinus of Valsalva with an interarterial course: case report and review of the literature. Neth Heart J. 2012;20: 463-71.

Key Words: pediatrics, congenital heart disease, anomalous origin of a coronary artery, cardiac magnetic resonance imaging 